
\title{
25 Research Square \\ The Expression Profiles and Role of Circular RNA in Peripheral Blood of Myocardial Infarction Patients
}

Qi Li

Department of Emergency Medicine

\section{Zhongjie Yu}

Institute for Translational Medicine

Mengyang Li

School of Basic Medicine

Zhe Su

Department of Emergency Medicine

Yuanyong Wang

Department of Thoracic Surgery

Jianxun Wang ( $\nabla$ wangjx@qdu.edu.cn )

School of Basic Medicine

Yi An ( $\square$ any2018@qdu.edu.cn )

Department of Cardiology

Yufang Gao ( $\square$ Gaoyufang0612@163.com )

The Affiliated Hospital of Qingdao University

\section{Research}

Keywords: Circular RNA, Myocardial Infarction, RNA sequencing, Biomarkers, Apoptosis

Posted Date: June 9th, 2020

DOI: https://doi.org/10.21203/rs.3.rs-33371/v1

License: (c) (i) This work is licensed under a Creative Commons Attribution 4.0 International License.

Read Full License 


\section{Abstract}

Background: Myocardial infarction (MI) is one of the most widespread illnesses seriously harmful to human health. However, the mechanisms of its pathogenesis have not yet been completely proved. circRNA is one kind of non-coding RNA, recently published data suggests that circRNAs play a crucial role in heart diseases, but their expression profiles and role in MI patients are not yet completely defined.

Methods: RNAs from peripheral blood samples were recruited for high-throughput RNA-seq analysis; The junction site part of circRNAs was confirmed by PCR with divergent primers; ROC curves were created to estimate the diagnostic value of circRNAs for Ml; The expression level of circRNAs in clinical and cell samples were detected by RT-qPCR; MiRNA target site in exons of circRNA loci was verified by using miRanda, The binding sites of miRNA and mRNA were predicted by RNAhybrid; The regulatory roles of circSLC8A1 in cardiomyocytes were detected by TUNEL and Immunoblotting.

Results: A total of 3862 circRNAs were detected to be remarkably changed, including 2738 circRNAs being upregulated, 1124 circRNAs being downregulated. circTMEM165, circUBAC2, circZNF609, circANKRD12, and circSLC8A1 were upregulated in MI and had closed loop structure. The results of ROC curves revealed that these five circRNAs had good sensitivity and specificity for MI diagnosis. Moreover, we created networks among circRNAs, miRNAs and mRNAs to predict the regulatory roles of circRNAs in MI. Besides that, we also demonstrated that circSLC8A1 contributed to cardiomyocytes apoptosis.

Conclusions: In this study, we depicted the expression profiles of circular RNA in peripheral blood of myocardial infarction patients, revealed the five circRNAs may be very beneficial for MI diagnosis and regarded as possible biomarkers. Moreover, we also revealed that circSLC8A1 played a critical role in cardiomyocytes apoptosis.

\section{Background}

Cardiovascular diseases (CVDs) are the most common cause of death globally. The number of death from cardiovascular disease accounted for about $1 / 3$ of all global death[1, 2]. MI is particularly common in CVDs and the major killer[3, 4]. Around $85 \%$ of deaths in CVDs are owing to $\mathrm{Ml}$ and stroke. $\mathrm{MI}$ is a multifactorial disease, which involved in complex pathogenesis[5]. MI is induced by partial or complete coronary artery suddenly blockaded or occlusion[6]. The consequence of blocked coronary arteries is an ischemic injury to the region of the heart[7]. Once the myocardial cell is damaged by ischemia, dead cardiomyocytes instead of fibroblasts, ultimately resulted in remodeling, ventricular malfunction, chronic heart failure, and even sudden cardiac death [8-12]. It is generally accepted that myocardial ischemia is irreversible after 12 hours of myocardial infarction, which is the key factor to impact on patient prognosis[6]. Hence, seeking inchoate and accurate biomarkers of $\mathrm{Ml}$ is urgently needed to improve diagnostic efficiency.

Recently, circRNAs have become focus studied and front field of RNA research based on the advanced RNA sequencing technology and bioinformatics analysis. circRNA is a kind of non-coding RNA, which 
contained a closed continuous loop structure and broadly expressed in multispecies genomes $[13,14]$. circRNAs were first reported years ago, but their functions in diseases are freshly appreciating and developing recently [15]. Most researchers reported that circRNAs are in charge of regulating gene expression principally by binding with and then resulting in miRNAs dysfunction[10]. A mount of reports verified the crucial functions of circRNAs in CVDs [16]. Abnormally expressed circRNAs were strongly linked to the occurrence and progress of CVDs, for instance, hypertrophy, heart failure and atherosclerosis $[17,18]$, etc. This plenty of evidence affords a novel orientation for discovering circRNAs as newfashioned biomarker for the diagnosis of CVDs.

To date, associations between circRNAs in peripheral blood and MI are not fully understood. Therefore, we explored the expression profiles of circRNA of MI patients and healthy controls by RNA-SEq. Besides, we measured the sensitivity of circRNAs for MI diagnosis, created networks among circRNAs-miRNAsmRNAs, and we also demonstrated the crucial role of circRNA in cardiomyocytes apoptosis.

\section{Methods}

All testing programs were performed according to protocols approved by the Medical Ethics Committee of the Affiliated Hospital of Qingdao University. All sample providers were informed of their blood samples. Clinical data were used for this study, and informed consents were signed.

\section{Sample collection}

Total of $50 \mathrm{MI}$ patients' peripheral blood samples were collected before percutaneous coronary intervention surgery between March 2018 and January 2019 in the affiliated hospital of Qingdao University (Qingdao, China). All patients were not treated with heparin, radiotherapy, or chemotherapy. The matched healthy controls were collected from the volunteers of the physical examination center. Fresh peripheral blood samples $(5 \mathrm{ml})$ form MI patients and healthy individuals were collected in EDTA tubes.

\section{Total RNA extraction}

The samples were used for RNA extraction via the Trizol method (Trizol-up reagents, TransGen, China). This experiment was performed according to the kit's recommendations. RNA samples were stored at $-80^{\circ} \mathrm{C}$.

\section{RNA quantification and qualification}

The purity of the extracted RNA was tested by a NanoPhotometer ${ }^{\circledR}$ spectrophotometer (IMPLEN, CA, USA). The RNA concentration was detected by Qubit ${ }^{\circledR}$ RNA Assay Kit in Qubit ${ }^{\circledR} 2.0$ Flurometer (Life Technologies, CA, USA). RNA integrity was measured by the RNA Nano 6000 Assay Kit of the Bioanalyzer 
2100 system (Agilent Technologies, CA, USA). RNA degradation and contamination were monitored on $1 \%$ agarose gels.

\section{Library preparation and circRNA sequencing}

A total of $5 \mu \mathrm{g}$ RNA per sample was used for the RNA library preparations. Firstly, rRNA was removed by Epicentre Ribozero $^{\mathrm{TM}}$ rRNA Removal Kit (Epicentre, USA) and ethanol precipitation. Subsequently, the linear RNA was digested with RNaseR (Epicentre, USA). The sequencing libraries were produced by NEBNext ${ }^{\circledR}$ Ultra $^{\text {TM }}$ Directional RNA Library Prep Kit for Illumina ${ }^{\circledR}$ (NEB, USA).

The cluster generation of the index-coded samples was performed on a cBot Cluster Generation System using TruSeq PE Cluster Kit v3-cBot-HS. After that, the libraries were sequenced on an Illumina Hiseq 4000 platform. These experiments were performed following the manufacturer's instruction and $150 \mathrm{bp}$ paired-end reads were generated.

\section{Data analysis and circRNA identification}

Firstly, clean data were acquired by wiping off reads containing adapter and low-quality reads from raw data. The clean data with high quality were used for downstream analyses. Index of the reference genome was created using Bowtie2 v2.2.8 and paired-end clean reads were united to the reference genome by using Bowtie[19]. The circRNA was detected and verified by using find_circ[20] and CIRI2[21]. Circos software was used to construct the circos figure.

\section{Divergent PCR}

The junction site part of circRNAs was confirmed by PCR with divergent primers. Convergent primers were invoked as the control. The products of the PCR amplification were authenticated by $1 \%$ agarose gel electrophoresis. The primers for circRNA validation were listed in supplementary data1 table 1. 


\begin{tabular}{|llll|}
\hline Variables, mean \pm SD or $\mathbf{n}$ & ctrl, $\mathbf{n}=\mathbf{5 0}$ & MIIn=50 & p-value \\
\hline Age (years) & $60.4 \pm 7.824$ & $63.7 \pm 9.772$ & 0.105 \\
\hline Gender (M/F) & $27 / 23$ & $30 / 20$ & 0.545 \\
\hline high(cm) & $166.71 \pm 8.564$ & $164.86 \pm 9.969$ & 0.394 \\
\hline weight(kg) & $69.89 \pm 18.005$ & $68.97 \pm 11.58$ & 0.795 \\
\hline BMI & $24.93 \pm 4.696$ & $25.4 \pm 3.849$ & 0.64 \\
\hline Systolic pressure & $128.25 \pm 16.505$ & $129.06 \pm 22.976$ & 0.865 \\
\hline Diastolic pressure & $72.64 \pm 8.735$ & $76.06 \pm 14.695$ & 0.236 \\
\hline Diabetes (yes/no) & $4 / 46$ & $10 / 40$ & 0.084 \\
\hline Smoking history (yes/no) & $16 / 34$ & $24 / 26$ & 0.102 \\
\hline Alcohol intake history (yes/no) & $10 / 40$ & $20 / 30$ & 0.029 \\
\hline Family history of CHD (yes/no) & $7 / 43$ & $18 / 32$ & 0.011 \\
\hline Other underlying diseases (yes/no) & $12 / 38$ & $15 / 35$ & 0.499 \\
\hline Pharmacological therapy (yes/no) & $11 / 39$ & $16 / 34$ & 0.26 \\
\hline TC (mmol/l) & $4.92 \pm 1.142$ & $4.21 \pm 1.063$ & 0.104 \\
\hline LDL-C (mmol/l) & $2.88 \pm 0.937$ & $2.55 \pm 0.589$ & 0.371 \\
\hline HDL-C (mmol/l) & $1.35 \pm 0.378$ & $0.99 \pm 1.82$ & 0.003 \\
\hline
\end{tabular}

Table 1

Detailed information of MI patient and control blood samples

\section{Real time-quantitative PCR}

Dysregulation circRNA reaffirmed was completed on a CFX96 Real-Time PCR Detection System (Bio-Rad). Total RNA was extracted using Trizol reagent. Reverse transcription reactions were performed by using the TransScript II One-Step gDNA Removal and cDNA Synthesis SuperMix (TransGen, China) to make cDNA according to the manufacturer's guide. TransStart Green qPCR SuperMix (TransGen) was used for Quantitative PCR (qPCR) analysis, the procedures were by the kit instructions. Levels of dysregulation circRNA analyzed by RT-qPCR were normalized to that of GAPDH[22]. The primers for RT-qPCR were listed in supplementary data 1 table 2 . 


\begin{tabular}{|lllllll|}
\hline circ_ID & chr & $\begin{array}{l}\text { full_ } \\
\text { length }\end{array}$ & $\begin{array}{l}\text { splice_ } \\
\text { length }\end{array}$ & $\begin{array}{l}\text { gene_ } \\
\text { name }\end{array}$ & $\begin{array}{l}\text { ML__ } \\
\text { readcount }\end{array}$ & $\begin{array}{l}\text { Control_ } \\
\text { readcount }\end{array}$ \\
\hline hsa_circ_0000826 & chr18 & 39618 & 994 & ANKRD12 & 816.599493 & 79.5731602 \\
\hline hsa_circ_0000896 & chr19 & 506 & 338 & FARSA & 446.180 .0157 & 72.0326233 \\
\hline hsa_circ_0000116 & chr1 & 3460 & 353 & MAN1A2 & 1933.94798 & 167.154168 \\
\hline hsa_circ_0000994 & chr2 & 1832 & 1832 & SLC8A1 & 313.178477 & 0 \\
\hline hsa_circ_0001445 & chr4 & 464 & 269 & SMARCA5 & 19590.5155 & 2907.27544 \\
\hline hsa_circ_0001414 & chr4 & 6372 & 585 & TMEM165 & 720.396765 & 102.724399 \\
\hline hsa_circ_0030720 & chr13 & 6198 & 358 & UBAC2 & 328.578223 & 24.0160633 \\
\hline hsa_circ_0001062 & chr2 & 181 & 181 & ZC3H6 & 516.545138 & 118.380163 \\
\hline hsa_circ_0000615 & chr15 & 874 & 874 & ZNF609 & 509.23034 & 0 \\
\hline
\end{tabular}

Table 2

The general information of circRNA

\section{Creation ROC curves}

ROC curves were created to estimate the diagnostic value of circRNAs for MI. A ROC curve was calculated and the specificity and sensitivity of predictive power were measured by the area under the curve (AUC), AUC was used to assess the diagnostic value of the circRNAs in the peripheral blood of MI patients[23]. SPSS24.0 and Graphpad prism7 were used for ROC curve calculating and drawing.

\section{GO and KEGG enrichment analysis}

Gene Ontology (GO) enrichment analysis for host genes of differentially expressed circRNAs were carried out by the GO seq R package, gene length bias was corrected during this procedure [24]. GO terms with a corrected $P$ value less than 0.05 were considered to be significantly enriched by differential expressed genes. As a database resource for understanding high-level functions and utilities of the biological system[25], KEGG is used to explore information from molecular-level (such as the cell, the organism, and the ecosystem). Especially largescale molecular datasets generated by genome sequencing and other high throughput experimental technologies (http://www.genome.jp/kegg/). We used KOBAS software to test the statistical enrichment of differential expression genes or circRNA host genes in KEGG pathways[26].

\section{Prediction the network of circRNAs-miRNAs-mRNAs}


circRNA can regulate the translation of mRNA via binding to a miRNA[10]. MiRNA target site in exons of circRNA loci was verified by using miRanda. The binding sites of miRNA and mRNA were predicted by RNAhybrid. Cytoscape was used to construct the circRNA-miRNA- mRNA networks.

\section{Cell culture and treatment}

AC16 cells were cultured in DMEM (Gibco) with $10 \%$ fetal bovine serum (TransGen), and $100 \mathrm{U} / \mathrm{ml}$ penicillin, $100 \mathrm{mg} / \mathrm{ml}$ streptomycin (Invitrogen) in a humidified $5 \% \mathrm{CO}_{2}$ incubator at $37^{\circ} \mathrm{C}$. When the cultured cells reached approximately $70 \%$ confluently, they were treated with $\mathrm{H}_{2} \mathrm{O}_{2}(100 \mu \mathrm{M})$ incubated at $37^{\circ} \mathrm{C}$ for 12 hours in the complete culture medium. The cells were collected for RNA extraction and dysregulated circRNA reconfirmation.

\section{Transfection}

We used Lipofectamine 3000 (Thermo Fisher) for transfection cirSLC8A1 siRNA (BGI) and overexpression vector (GENE Chem). The procedures were in accordance with the kit instructions.

\section{Mitochondrial staining and analysis of mitochondrial fission}

Cells were plated onto the poly-L-lysine coated coverslips. After treatment, they were stained for 30 minutes with $0.02 \mu \mathrm{M}$ MitoTracker Red at $37^{\circ} \mathrm{C}$. Mitochondria were imaged using a laser-scanning confocal microscope (Zeiss LSM510 META).

\section{Apoptosis assays}

Apoptosis was determined by the terminal deoxynucleotidyl transferase-mediated dUTP nick-endlabelling (TUNEL) using a kit from TransGen. The detection procedures were in accordance with the kit instructions.

\section{Immunoblotting}

Cells were lysed for 20 minutes on ice in RIPA lysis buffer containing a protease inhibitor cocktail and DMSF. The samples were subjected to $12 \%$ SDS-PAGE and transferred to nitrocellulose membranes. Blots were probed with primary antibodies anti-Caspase3 (Abcom, 1:1000), anti-Cyto $c$ (Abclone, 1:1000) antiTubulin (Affinity, 1:1000) anti- $\beta$-actin (TransGen, 1:2000) at $4^{\circ} \mathrm{C}$ overnight with gently shaking. After three times washing with PBS, the horseradish peroxidase (HRP)-conjugated secondary antibodies were added. Antigen antibody complexes were visualized by enhanced chemiluminescence. Enhanced ECL TM prime detection reagent (GE) used to visualize antigen-antibody complexes, the density quantified by Image J. 


\section{Statistical analysis}

The results are expressed as the mean \pm SEM of at least three independent experiments. The statistical comparison between different groups was completed by one-way analysis of variance (ANOVA) for multiple comparisons or t-test with Welch's correction for two groups. Statistical analyses were completed with GraphPad Prism 7.0 (GraphPad Software, Inc., San Diego, CA). $P<0.05$ was regarded as statistically significant.

\section{Results}

\section{The landscape of circRNA expression profiles in peripheral blood from Ml patients and healthy individuals}

The circRNAs were identified by find_circ and Bowtie2 based on high-quality clean data. A total of 24686 circRNAs were recognized in five pairs of $\mathrm{Ml}$ and healthy controls samples. The size of the circRNAs extended from $\approx 100$ to $2500 \mathrm{nt}$ (Fig. 1a). Differentially expressed circRNAs were analyzed by DEGseq, results were considered statistically significant when padj $<0.05$. The volcano and scatter plots displayed the variation in circRNA expression between MI and control (Fig. 1b, c). Hierarchical clustering heatmap analysis is shown in Fig. 1d illustrates a unique circRNA expression landscape among MI and healthy individuals. Our results presented that there was an obvious difference of circRNA expression profile between the $\mathrm{Ml}$ and healthy controls. Consequently, 3862 circRNAs were altered in the MI patients. There were 2738 circRNAs up-regulated and 1124 were down-regulated in the MI group compared with the healthy controls. Between the distinguishable expressed circRNAs, $89 \%$ are exonic, $10 \%$ are intronic, and $1 \%$ belong to intergenic (Fig. 1e). As showed in Fig. 1f, the transcription of dysregulated circRNAs was observed to be broadly dispersed in all chromosomes, including, chr1, chr2, chr3, chr4, chr5, chr6, chr7, chr8, chrX, and chr9 are the top ten. Top 50 differentially expressed circRNAs are depicted in supplementary data 2 .

\section{circRNAs screening and circular structure identification}

Firstly, we screened candidate circRNAs by comparing MI RNA-seq data with human heart failing RNA-seq data[27]. Then we detected the circular structure of intersection circRNAs (supplementary Fig. 1a, Table.2). We tested the expression of intersection circRNAs by adding them from the cDNA of myocardial cells by using the divergent primer. Additionally, added products of circRNAs were sequenced by using Sanger-sequencing to further confirm the circularized junction size of the circRNAs (Fig. 2). Lastly, we confirmed that circTMEM165, circUBAC2, circZNF609, circANKRD12, and circSLC8A1 were derived from transcripts and had closed loop structures.

\section{Authentication of differentially expressed circRNAs}


To verify the RNA-seq data, five intersectional circRNAs (circTMEM165, circUBAC2, circZNF609, circANKRD12, circSLC8A1) were selected and validated in clinical samples by using RT-qPCR $(n=50$, the basic information of clinical samples was described in Table 1). The results revealed that the expression of these five circRNAs was significantly up-regulated in MI patients (Fig. 3). The RT-qPCR data was strongly consistent with the circRNA sequencing results.

To reconfirm the dysregulation of these circRNAs in the cell model, we created cardiomyocytes injury model induced by reactive oxygen species (ROS) to simulate cell damage during MI[10]. The data of the cell model showed that all the validated circRNAs were up-regulated with prolonging stimulation time. In these five circRNAs, the expression level of circSLC8A1 was the most change a great deal (supplementary Fig. 2). These results were highly consisted of the data of clinical samples. Collectively, these two results verified the accuracy and repeatability of the circRNA sequencing results.

\section{Potential diagnostic values of circRNAs for MI patients}

To evaluate the potential diagnostic value of selected circRNAs for MI, we computed and created ROC curves (Fig. 4) for RT-qPCR-validated circRNAs to discriminate MI patients from healthy individuals according to the fold changes of these circRNAs expressions. The results revealed that the AUC of these circRNAs at least reached 0.713 , especially, the AUC of recombination circRNAs reached up to 0.9236 (Table. 3), indicating that these circRNAs expressed in peripheral blood could set apart the MI patients from the healthy individuals. Our data discovered the potential values of circRNAs MI diagnosis.

\begin{tabular}{|llll|}
\hline circRNA & AUC & $\begin{array}{l}\text { Validation Patients(n=50) } \\
\text { 95\% Confidence Interval }\end{array}$ & P value \\
\hline circTMEM165 & 0.713 & 0.6137 to 0.8123 & 0.0002442 \\
\hline circUBAC2 & 0.7512 & 0.6536 to 0.8488 & $<0.0001$ \\
\hline circZNF609 & 0.902 & 0.8407 to 0.9633 & $<0.0001$ \\
\hline circSLC8A1 & 0.8004 & 0.7141 to 0.8867 & $<0.0001$ \\
\hline circANKRD12 & 0.7152 & 0.6160 to 0.8144 & 0.0002104 \\
\hline circRNAs combination & 0.9236 & 0.8705 to 0.9767 & $<0.0001$ \\
\hline
\end{tabular}

Table 3

AUC of RT-qPCR-validated circRNAs in validation patients

\section{Enrichment analysis of circRNAs host genes}


The source genes of the validated differentially expressed circRNAs were uncovered by using GO annotation, as showed in supplementary Fig. 3a. The most remarkably improved GO terms about the biological process (BP), cellular component (CC), and molecular function (MF) classes were cellular macromolecule metabolic process (GO:0044260, $P=2.892 \mathrm{E}-46$ ), intracellular part (GO:0044424, $P=$ 5.136E-69), and protein binding (G0:0005515, $P=8.9672 \mathrm{E}-34)$, respectively. The data of KEGG pathway study of the circRNAs genes are shown in supplementary Fig. 3b. The most relevant genes of differentially expressed circRNAs are ubiquitin mediated proteolysis (rich factor $=0.328467153$, q value $=$ $1.56 \mathrm{E}-06$, gene number $=45$ ).

\section{Predicted circRNA-miRNA-mRNA regulatory network}

miRNA binding sites exist in circRNAs, which can function as miRNA sponges. To further explore their possible roles in MI patients, we predicted the circRNA-miRNA-mRNA network and analyzed by using miRanda and RNAhybrid. The top five predicted miRNAs for these up-regulated circRNAs were presented in Table 4, the target mRNAs of miRNAs are listed in supplementary data 3. Additionally, circRNA-miRNA targeted mRNA networks are also constructed for showing regulatory relationships between them (Fig. 5). 
Table 4

The predicted miRNAs of circRNAs

\begin{tabular}{|ll|}
\hline Up-regulated circRNAs & Predicted miRNAs \\
\hline circTMEM165 & hsa-miR-103b \\
\hline hsa-miR-1255b-2-3p & hsa-miR-1323 \\
\hline hsa-miR-548a-3p \\
\hline circZNF609 & hsa-miR-1251-5p \\
\hline hsa-miR-644a \\
\hline hsa-miR-4303 \\
\hline hsa-miR-221-5p \\
\hline hsa-miR-1260b \\
\hline hsa-miR-1247-5p \\
\hline hsa-miR-876-5p \\
\hline hsa-miR-452-3p \\
\hline hsa-miR-3167 \\
\hline circANKRD12 & hsa-miR-143-3p \\
\hline hsa-miR-130b-5p \\
hsa-miR-200b-3p \\
\hline hsa-miR-2115-5p \\
\hline hsa-miR-509-5p \\
\hline hsa-miR-519d-5p \\
\hline hsa-miR-539-3p \\
\hline hsa-miR-1202 \\
\hline hsa-miR-27a-3p \\
\hline hsa-miR-27b-3p \\
\hline hsa-miR-30d-3p \\
\hline
\end{tabular}

Moreover, we noted the potential function of these regulatory networks. For example, circTMEM165 is predicted as 'sponge' to hsa-miR-548a-3p, which can bind to mRNA GREM1, which is responsible for 
regulating organogenesis, body patterning, and tissue differentiation; The network of circZNF609- hsamiR-221-5p-WDR7 might be involved in the low-density lipoprotein (LDL) metabolism; The regulatory pathway of circANKRD12-hsa-miR-876-5p-MYLK may promote myosin interaction with actin and facilitate to produce contractile activity; circANKRD12-hsa-miR-130b-5p targets both ACSM2A and RNF213, which regulates mitochondrial acyl-coenzyme A synthetase and ATPase activity, respectively. These networks indicate possible interactional RNA regulatory relationships between circRNAs, miRNAs, and mRNAs in MI.

\section{The role of circRNA in cardiomyocyte apoptosis}

In the early stages of Ml, hypoxia caused by cardiomyocytes apoptosis[28]. circRNA was a new noncoding RNA, which was reported could regulate apoptosis induced by hypoxia[29]. In our present study, we found that the expression level of circSLC8A1 was significantly changed in both MI patients and cell injury model (Fig. 3, supplementary Fig. 2). This finding suggested circSLC8A1 might play a critical role in cardiomyocytes injury. To verify our hypothesis, we firstly knocked down the expression level of circSLC8A1 by using circSLC8A1 siRNA, and then cardiomyocytes were treated with $100 \mu \mathrm{M} \mathrm{H}_{2} \mathrm{O}_{2}$. Our results revealed that losing circSLC8A1 could inhibit mitochondrial fission and the release of cytochrome $C$, and then protect cardiomyocytes against $\mathrm{H}_{2} \mathrm{O}_{2}$-induced apoptosis (Fig. 6). Also, we also overexpressed circSLC8A1 in cardiomyocytes by using plasmid. These data indicated that circSLC8A1 could enhance the sensitivity of cardiomyocytes to $\mathrm{H}_{2} \mathrm{O}_{2}$, and promote mitochondrial fission and the release of cytochrome $c$, and then contributes to cell apoptosis induced by $\mathrm{H}_{2} \mathrm{O}_{2}$ (supplementary Fig. 4). Taken together, our results demonstrated circSLC8A1 played a key role in cardiomyocytes apoptosis.

\section{Discussion}

$\mathrm{Ml}$ is a kind of multifactorial disease, which causes complex pathogenesis and leads to high mortality. It's difficult to diagnose and prompt intervention $\mathrm{Ml}$ before the disease occurs. As a consequence, there is imperative that early detection markers for MI diagnose. circRNAs are single-stranded, covalently closed molecules[30], which are expressed in many species[20,31]. The application of advanced RNA-seq technology has improved our understanding of knowledge about the specific circRNA expression profiles in human diseases[13]. Some researchers noted that circRNAs may serve as a novel kind of ideal biomarker for disease diagnosis[32]. In our present study, circRNA-seq was employed to distinguish differences expression profile of circRNA in peripheral blood between MI patients and healthy individuals, our results revealed the possible contribution of differentially expressed circRNAs in MI pathology.

Recent reports have demonstrated that circRNAs are more constant, varied, and conserved when compared with other kinds of RNAs, including mRNAs, miRNAs, and IncRNAs[20]. circRNAs are extensively expressed in multi-species[20,31] and involved in disease occurrence. These characteristics of circRNA may endow them to be a new kind of biomarker for disease diagnosis. In our present study, to detect the novel and original biomarkers of $\mathrm{Ml}$, we firstly verified the expression landscape of circRNAs in 
peripheral blood between five MI patients and five healthy individuals by using RNA-sEq. There were 2738 up-regulated circRNAs and 1124 down-regulated circRNAs in the MI patients compared with the healthy controls. After compared our sequencing data with others, five circRNAs (circTMEM165, circUBAC2, circZNF609, circANKRD12, circSLC8A1) were selected and further reaffirmed in MI patients and cell injury models by RT-qPCR, the results strongly consisted with the sequencing data.

Early diagnosis can guide clinical medication to effectively treat $\mathrm{MI}$ and significantly improved their prognosis. circRNAs have already been confirmed as effective biomarkers for numerous diseases, such as cancer[33], acquired pneumonia[34], Kawasaki disease[35]. It was reported that hsa_circ_0004104 was upregulated in coronary artery disease (CAD), and its overexpression might contribute to the pathogenesis of CAD[36]. Hence, circRNA also should enhance the diagnosis of MI. In this study, our data revealed that thousands of circRNAs were observably dysregulated in peripheral blood from MI patients, circTMEM165, circUBAC2, circZNF609, circANKRD12, and circSLC8A1 were selected for further study. As we expected, these five circRNAs reconfirmed as being remarkedly upregulated in the peripheral blood of $50 \mathrm{Ml}$ patients by RT-qPCR. The AUC of these five circRNA were $0.713,0.7512,0.902,0.8004,0.7152$, respectively. When we combinational analyzed this five circRNA, the combinational AUC was even up to 0.9236 . Our data indicated these circRNAs might be significantly associated with $\mathrm{Ml}$ and has the potential to assist in the diagnosis of MI.

In our study, circRNA-seq data were used to predict circRNA-miRNA-mRNA networks and aimed to find the possible roles of circTMEM165, circUBAC2, circZNF609, circANKRD12, and circSLC8A1 in MI. We observed that the possible target mRNAs of these circRNAs including GREM1, MYLK, ACSM2A and RNF213, which played a key role in regulating organogenesis[37], tissue differentiation[38], LDL metabolism[39], myosin interaction with actin[40], mitochondrial acyl-coenzyme A synthetase and ATPase activity[41], respectively. All of them are highly correlated to CVDs.

Recently, several pieces of research have indicated that circRNAs might be key regulators in CVDs. Wu et al. [35] showed that the levels of circ_ANRIL and hsa_circ_0123996 in the serum from Kawasaki patients were markedly different from those in healthy controls, and the expression level will be changed after therapy. Wang et al. [36] reported that hsa_circ_0004104 was markedly upregulated in CAD patients, overexpression of hsa_circ_0004104 contributed to the dysregulation of atherosclerosis-related genes. All these pieces of evidence powerfully support the view that circRNAs play crucial roles in CVDs occurrence and progression. In our present study, we found that circSLC8A1 is the most remarkably changed among these five candidate circRNAs. After functional verification, our results revealed that the expression level of circSLC8A1 was highly associated with cardiomyocytes apoptosis, which may contribute to accelerate the development of MI. But the detailed mechanism still needs further explored.

\section{Conclusion}

In our present study, we reported the expression profiles of circular RNA in the peripheral blood of MI patients. Bioinformatics analysis and RT-qPCR verification revealed that the five circRNA (circTMEM165, 
circUBAC2, circZNF609, circANKRD12, and circSLC8A1) were highly correlated to MI. Moreover, all of these five circRNAs may be very beneficial for MI diagnosis and regarded as possible biomarkers. Meanwhile, these five circRNAs could be involved in the regulation of hundreds of mRNAs, and numerous of these mRNAs may affect the occurrence of MI. Besides that, we also demonstrated that circSLC8A1 contributed to cardiomyocytes apoptosis.

\section{List Of Abbreviations}

MI, Myocardial infarction; circRNA, Circular RNA; qRT-PCR, Quantitative real-time polymerase chain reaction; ROC, Receiver operating characteristic; GO, Gene Ontology; KEGG, Kyoto Encyclopedia of Genes and Genomes; miRNA, microRNA; CVDs, Cardiovascular diseases; RNA-Seq, high-throughput RNA sequencing; EDTA, Ethylenediaminetetraacetic acid; qPCR, Quantitative PCR; AUC, the area under the curve; ROS, reactive oxygen species; BP, biological process; CC, cellular component; MF, molecular function; LDL, low-density lipoprotein; CAD, artery disease.

\section{Declarations}

\section{Ethics approval and consent to participate:}

All testing programs were performed according to protocols approved and consented by the Medical Ethics Committee of the Affiliated Hospital of Qingdao University (NO. QYFY WZLL2561).

\section{Consent for publication:}

All sample providers were informed of their blood samples. Clinical data were used for this study, and informed consents were signed. All of them consented for publication.

\section{Availability of data and materials:}

The datasets used and/or analyzed during the current study are available from the corresponding author on reasonable request.

\section{Competing interests:}

The authors declare no conflicts of interest.

\section{Funding:}

Not applicable 


\section{Authors' contributions:}

Qi Li, Jianxun Wang, Yi An and Yufang Gao generate the idea;

Qi Li, Zhongjie Yu, Mengyang Li, Zhe Su, Yuanyong Wang perform experiment;

Qi Li and Zhongjie Yu prepare and edit the manuscript.

\section{Acknowledgements:}

This work was supported by the National Science Foundation of China (grant numbers 81071246 ). This work was supported by the Qingdao Minsheng Science and Technology Plan Project (grant numbers 186-1-80-nsh).

\section{References}

1. T M, K G, F S, P S, GI S, C D, W M, ME K, S L: Cardiovascular mortality attributable to dietary risk factors in 51 countries in the WHO European Region from 1990 to 2016: a systematic analysis of the Global Burden of Disease Study. European journal of epidemiology 2019, 34:37-55.

2. $R$ M, $P$ L-S, P S, S E, E V, $P$ O, M M: Trends in premature avertable mortality from non-communicable diseases for 195 countries and territories, 1990-2017: a population-based study. The Lancet Global health 2020, 8:e511-e523.

3. Anderson JL, Morrow DA: Acute Myocardial Infarction. N Engl J Med 2017, 376:2053-2064.

4. None.: Cardiovascular disease in Europe 2016: an epidemiological update. European Heart Journal 2016, 37:3182-3183.

5. Kontou P, Pavlopoulou A, Braliou G, Bogiatzi S, Dimou N, Bangalore S, Bagos P: Identification of gene expression profiles in myocardial infarction: a systematic review and meta-analysis. $B M C M e d$ Genomics 2018, 11:109.

6. Wu D, Zhang K, Hu P: The Role of Autophagy in Acute Myocardial Infarction. Front Pharmacol 2019, 10:551.

7. Swirski FK, Nahrendorf M: Cardioimmunology: the immune system in cardiac homeostasis and disease. Nat Rev Immunol 2018, 18:733-744.

8. Gabriel-Costa D: The pathophysiology of myocardial infarction-induced heart failure. Pathophysiology 2018, 25:277-284.

9. Bougouin W, Marijon E, Puymirat E, Defaye P, Celermajer DS, Le Heuzey JY, Boveda S, Kacet S, Mabo $P$, Barnay $C$, et al: Incidence of sudden cardiac death after ventricular fibrillation complicating acute myocardial infarction: a 5-year cause-of-death analysis of the FAST-MI 2005 registry. Eur Heart $J$ 2014, 35:116-122. 
10. Li M, Ding W, Tariq MA, Chang W, Zhang X, Xu W, Hou L, Wang Y, Wang J: A circular transcript of ncx1 gene mediates ischemic myocardial injury by targeting miR-133a-3p. Theranostics 2018, 8:5855-5869.

11. Gyongyosi M, Winkler J, Ramos I, Do QT, Firat H, McDonald K, Gonzalez A, Thum T, Diez J, Jaisser F, et al: Myocardial fibrosis: biomedical research from bench to bedside. Eur J Heart Fail 2017, 19:177191.

12. Ezekowitz JA, Kaul P, Bakal JA, Armstrong PW, Welsh RC, McAlister FA: Declining in-hospital mortality and increasing heart failure incidence in elderly patients with first myocardial infarction. $J$ Am Coll Cardiol 2009, 53:13-20.

13. Hsiao KY, Sun HS, Tsai SJ: Circular RNA - New member of noncoding RNA with novel functions. Exp Biol Med (Maywood) 2017, 242:1136-1141.

14. Jens M: Dissecting Regulatory Interactions of RNA and Protein. Springer Theses 2014:69-80.

15. Sanger HL, Klotz G, Riesner D, Gross HJ, AK. K: Viroids are single-stranded covalently closed circular RNA molecules existing as highly base-paired rod-like structures. . Proc Natl Acad Sci U S A 1976, 73:3852-3856.

16. Cai L, Qi B, Wu X, Peng S, Zhou G, Wei Y, Xu J, Chen S, Liu S: Circular RNA Ttc3 regulates cardiac function after myocardial infarction by sponging miR-15b. J Mol Cell Cardiol 2019, 130:10-22.

17. Wang K, Long B, Liu F, Wang JX, Liu CY, Zhao B, Zhou LY, Sun T, Wang M, Yu T, et al: A circular RNA protects the heart from pathological hypertrophy and heart failure by targeting miR-223. Eur Heart $J$ 2016, 37:2602-2611.

18. Holdt LM, Stahringer A, Sass K, Pichler G, Kulak NA, Wilfert W, Kohlmaier A, Herbst A, Northoff BH, Nicolaou A, et al: Circular non-coding RNA ANRIL modulates ribosomal RNA maturation and atherosclerosis in humans. Nat Commun 2016, 7:12429.

19. Langmead B, Trapnell C, Pop M, Salzberg SL: Ultrafast and memory-efficient alignment of short DNA sequences to the human genome. Genome Biol 2009, 10:R25.

20. Memczak S, Jens M, Elefsinioti A, Torti F, Krueger J, Rybak A, Maier L, Mackowiak SD, Gregersen LH, Munschauer $\mathrm{M}$, et al: Circular RNAs are a large class of animal RNAs with regulatory potency. Nature 2013, 495:333-338.

21. Gao Y, Zhang J, Zhao F: Circular RNA identification based on multiple seed matching. Brief Bioinform 2018, 19:803-810.

22. Lei B, Zhou J, Xuan X, Tian Z, Zhang M, Gao W, Lin Y, Ni B, Pang H, Fan W: Circular RNA expression profiles of peripheral blood mononuclear cells in hepatocellular carcinoma patients by sequence analysis. Cancer Med 2019, 8:1423-1433.

23. Liu XX, Yang YE, Liu X, Zhang MY, Li R, Yin YH, Qu YQ: A two-circular RNA signature as a noninvasive diagnostic biomarker for lung adenocarcinoma. J Trans/ Med 2019, 17:50.

24. Young MD, Wakefield MJ, Smyth GK, Oshlack A: Gene ontology analysis for RNA-seq: accounting for selection bias. Genome Biol 2010, 11:R14. 
25. Kanehisa M, Araki M, Goto S, Hattori M, Hirakawa M, Itoh M, Katayama T, Kawashima S, Okuda S, Tokimatsu T, Yamanishi Y: KEGG for linking genomes to life and the environment. Nucleic Acids Res 2008, 36:D480-484.

26. Mao X, Cai T, Olyarchuk JG, Wei L: Automated genome annotation and pathway identification using the KEGG Orthology (KO) as a controlled vocabulary. Bioinformatics 2005, 21:3787-3793.

27. Werfel S, Nothjunge S, Schwarzmayr T, Strom TM, Meitinger T, Engelhardt S: Characterization of circular RNAs in human, mouse and rat hearts. J Mol Cell Cardiol 2016, 98:103-107.

28. Y L, S R, J X, Y W, Y X: EIF4A3-Induced circ-BNIP3 Aggravated Hypoxia-Induced Injury of H9c2 Cells by Targeting miR-27a-3p/BNIP3. Molecular therapy Nucleic acids 2020, 19:533-545.

29. RY D, FL L, Y L: Circular RNA hsa_circ_0010729 regulates vascular endothelial cell proliferation and apoptosis by targeting the miR-186/HIF-1a axis. Biochemical and biophysical research communications 2017, 490:104-110.

30. Jeck WR, Sharpless NE: Detecting and characterizing circular RNAs. Nat Biotechnol 2014, 32:453461.

31. Jeck WR, Sorrentino JA, Wang K, Slevin MK, Burd CE, Liu J, Marzluff WF, Sharpless NE: Circular RNAs are abundant, conserved, and associated with ALU repeats. RNA 2013, 19:141-157.

32. Meng S, Zhou H, Feng Z, Xu Z, Tang Y, Li P, Wu M: CircRNA: functions and properties of a novel potential biomarker for cancer. Mol Cancer 2017, 16:94.

33. Li X, Wang Y, Han C, Li P, Zhang H: Colorectal cancer progression is associated with accumulation of Th17 lymphocytes in tumor tissues and increased serum levels of interleukin-6. Tohoku J Exp Med 2014, 233:175-182.

34. Zhao T, Zheng Y, Hao D, Jin X, Luo Q, Guo Y, Li D, Xi W, Xu Y, Chen Y, et al: Blood circRNAs as biomarkers for the diagnosis of community-acquired pneumonia. J Cell Biochem 2019.

35. Wu J, Zhou Q, Niu Y, Chen J, Zhu Y, Ye S, Xi Y, Wang F, Qiu H, Bu S: Aberrant expression of serum circANRIL and hsa_circ_0123996 in children with Kawasaki disease. J Clin Lab Anal2019, 33:e22874.

36. Wang L, Shen C, Wang Y, Zou T, Zhu H, Lu X, Li L, Yang B, Chen J, Chen S, et al: Identification of circular RNA Hsa_circ_0001879 and Hsa_circ_0004104 as novel biomarkers for coronary artery disease. Atherosclerosis 2019, 286:88-96.

37. A Z, F L, J L-R, C K, N M, R Z: Conserved cis-regulatory regions in a large genomic landscape control SHH and BMP-regulated Gremlin1 expression in mouse limb buds. BMC developmental biology 2012, 12:23.

38. Y M, K T, S A, T U, Y S, M P, GM S, W R, W V: Absolute expressions of hypoxia-inducible factor-1 alpha (HIF1A) transcript and the associated genes in chicken skeletal muscle with white striping and wooden breast myopathies. PloS one 2019, 14:e0220904.

39. A K, F M, ID K, M M, O K, M P: Genome-wide DNA Methylation Profiling of Blood from Monozygotic Twins Discordant for Myocardial Infarction. In vivo (Athens, Greece) 2020, 34:361-367. 
40. SR K, C B, D C, P G, M L, PF P, X J, N B-N: Exome sequencing in seven families and gene-based association studies indicate genetic heterogeneity and suggest possible candidates for fibromuscular dysplasia. Journal of hypertension 2015, 33:1802-1810; discussion 1810.

41. H K, Y M, T H, H O, H S, T M, H I, M S, D T, KH H, et al: Biochemical and Functional Characterization of RNF213 (Mysterin) R4810K, a Susceptibility Mutation of Moyamoya Disease, in Angiogenesis In Vitro and In Vivo. Journal of the American Heart Association 2015, 4.

\section{Figures}


$\mathbf{a}$

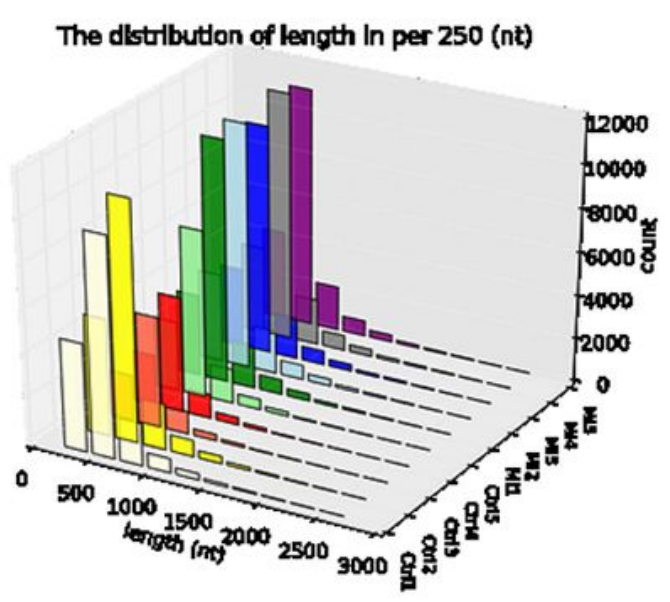

c

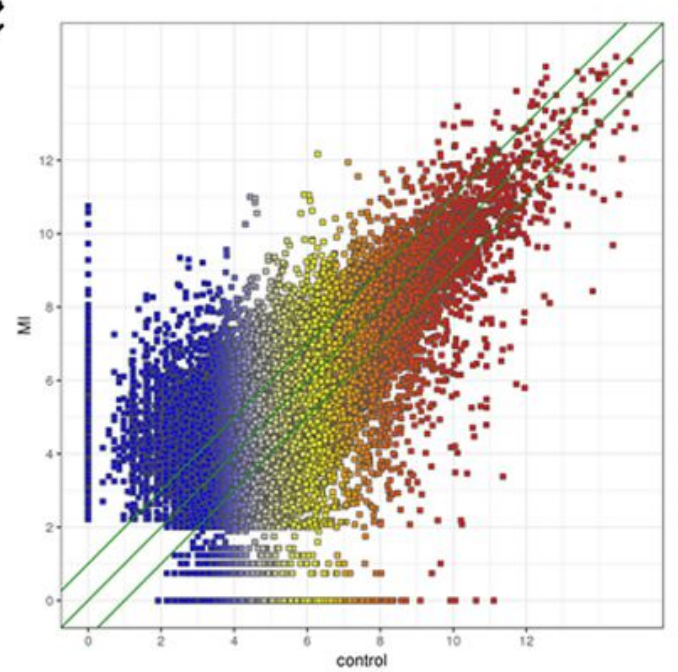

$\mathbf{e}$

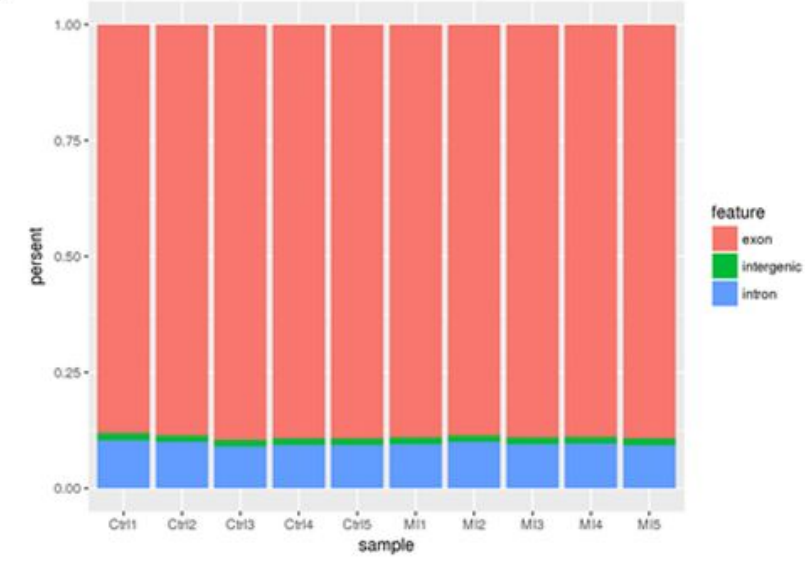

b

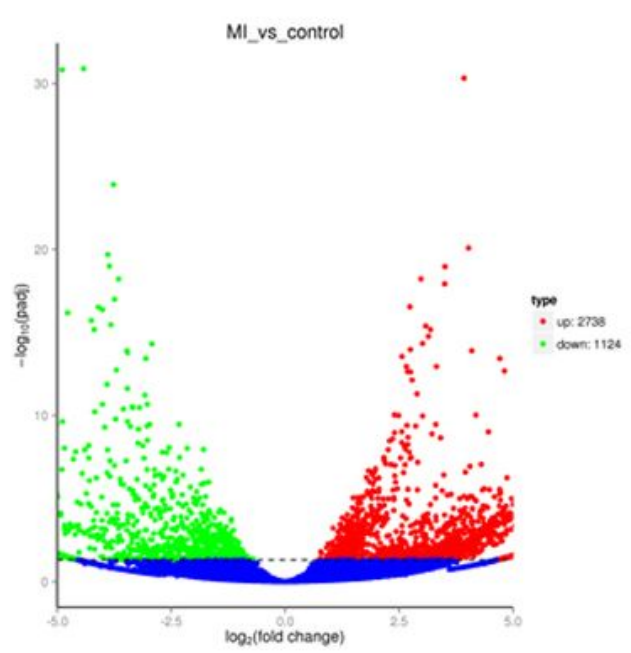

d

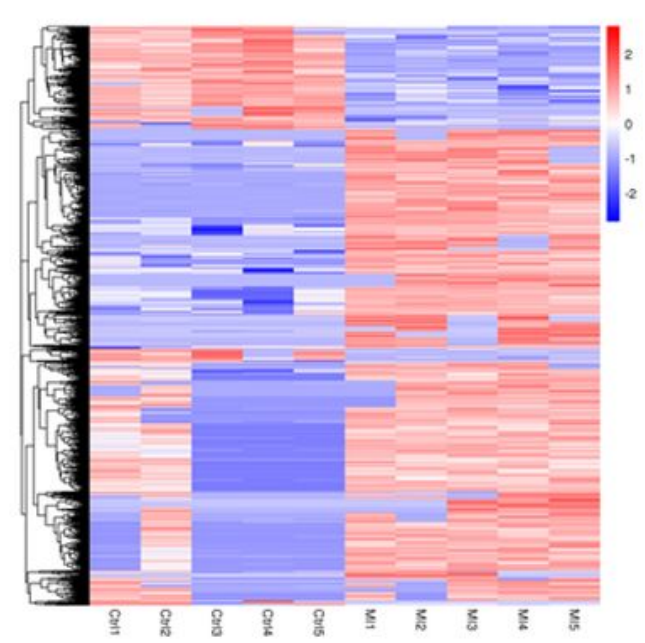

f

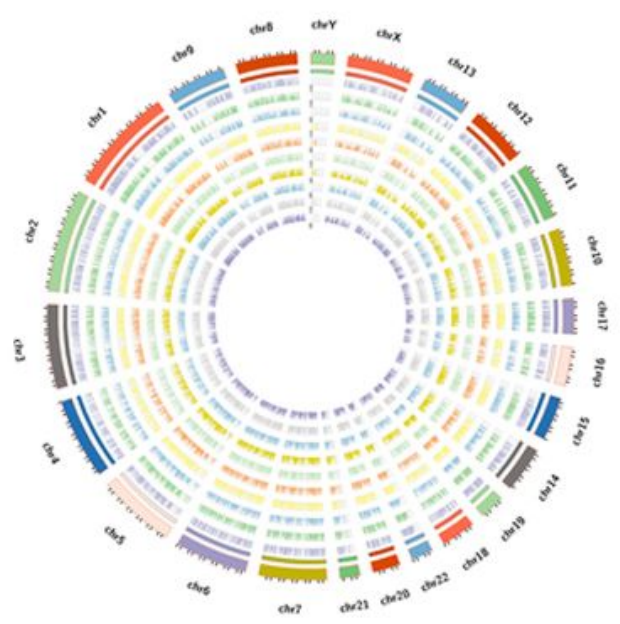

\section{Figure 1}

Landscape of circRNA profiles. (a) Length distribution of circRNAs. (b) Volcano plot of differentially expressed circRNAs in Ml. The abscissa represents the fold change of circRNA in different groups, and the ordinate represents the level of statistical significance. The plots in the figure represents circRNAs. The blue points denote no significant difference, red points denote the expression level of circRNAs are significantly up-regulation, green points denote the expression level of circRNAs are significantly down- 
regulation. (c) The scatter plot presents the circRNA expression variations between the $\mathrm{MI}$ and paired healthy controls. The nodal increment of the $x$ and $y$ axes are $1(\log 2 \mathrm{FC}=1)$. The circRNAs above the top green line and below the bottom green line indicate greater than 2-fold change $(T P M>2)$ between the $M I$ and control. (d) Hierarchical cluster analysis shown the differentially expression profiles circRNAs among MI samples and normal samples. The up- and down-regulated circRNA are colored in red and blue, respectively. Ctrl1-Ctrl5 represent healthy controls, and MI1-MI5 represent MI patients. (e) Histogram shown the percentage of circRNA from different genomic origin. (f) Pie chart shown differentially expressed circRNAs based on Chromosomal distributions.

a

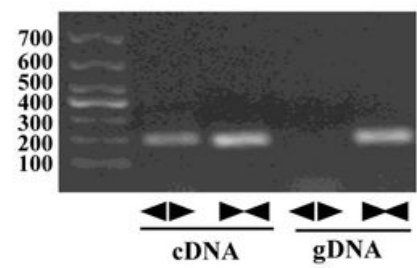

circTMEM165 Exon

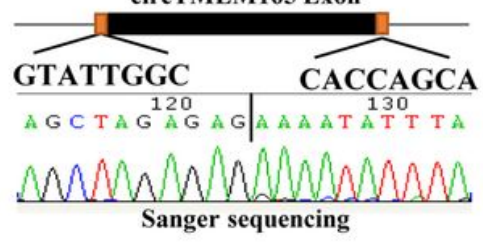

b

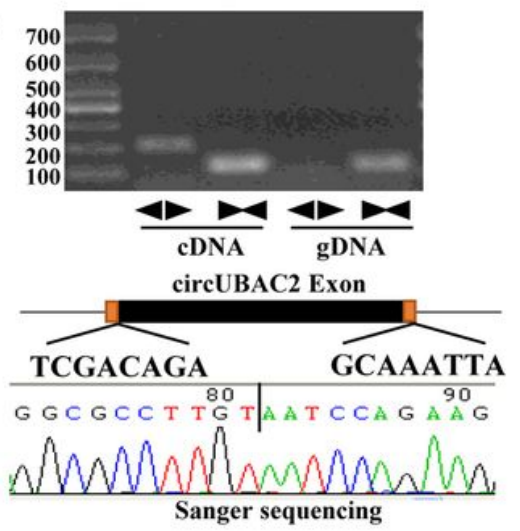

c

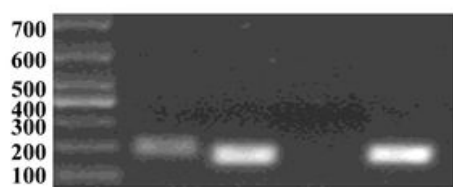

$\frac{1 \longrightarrow}{\text { cDNA }} \frac{\longrightarrow}{\text { gDNA }}$ circZNF609 Exon

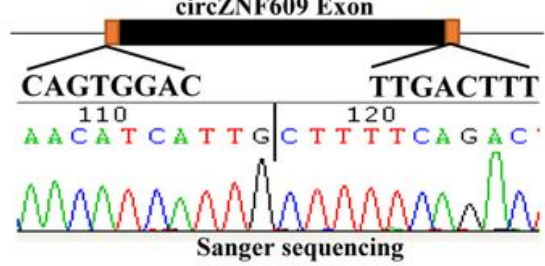

d

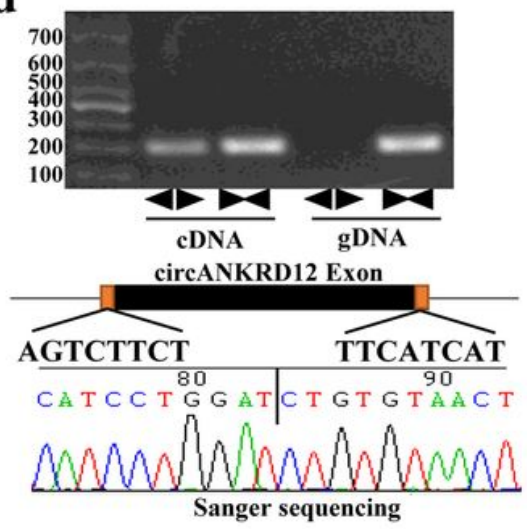

e
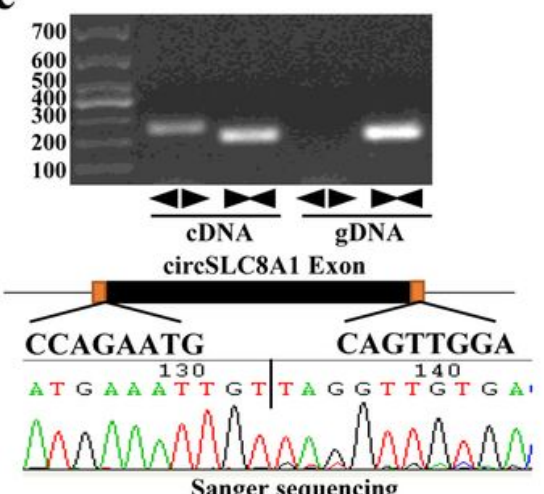

Sanger sequencing

\section{Figure 2}

Validation the circular structure of circRNA. circRNAs exist in human myocardial cell line (AC16). Clear single bands were amplified from the CDNA of AC16 by divergent primers, while they could not be amplified from gDNA. Sanger-Seq validated the head-to-tail junction of circRNAs. 
$\mathbf{a}$

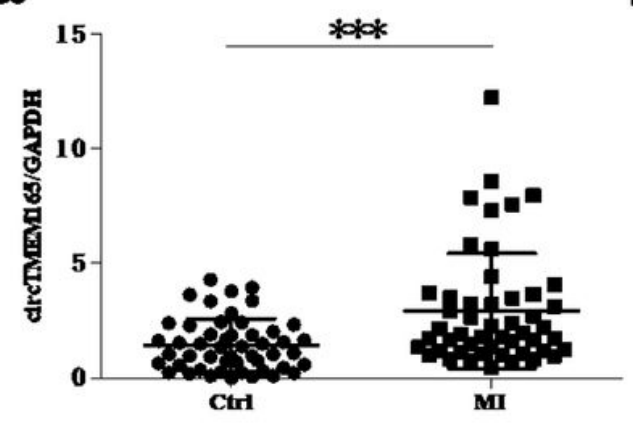

d

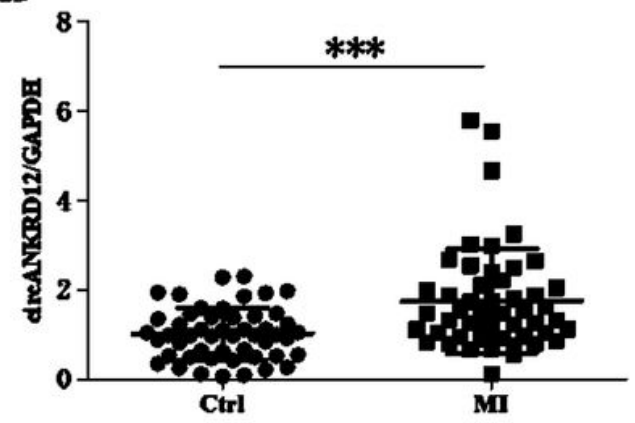

C
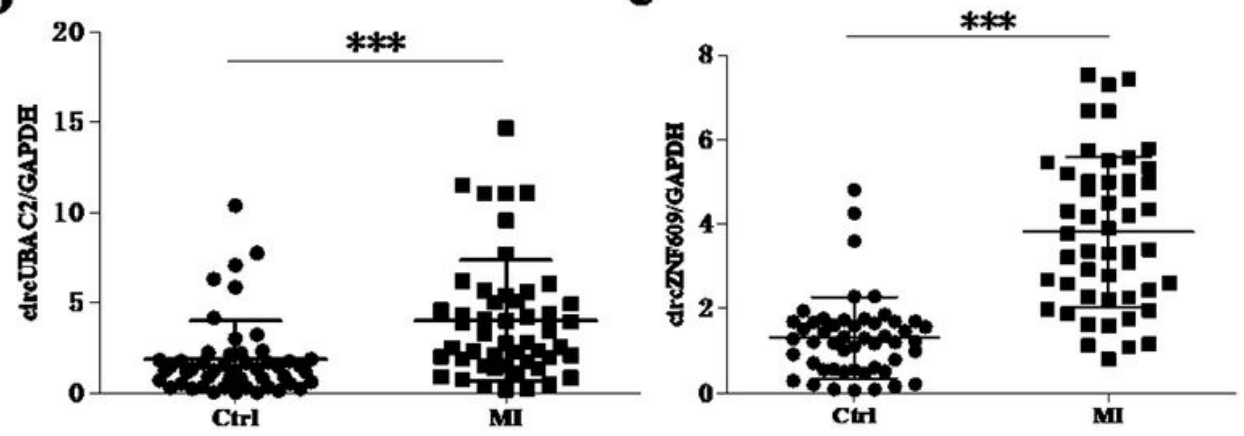

$\mathbf{e}$

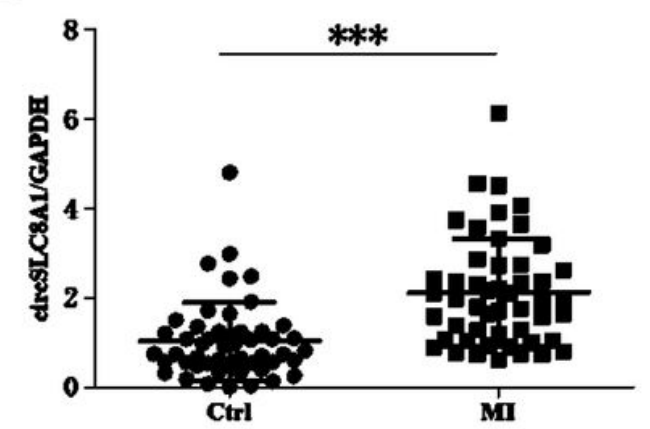

Figure 3

Validation of differentially expressed circRNAs. RT-qPCR validation of selected circRNAs. (a-e) The relative expression levels of circRNAs in peripheral blood MI patients and controls. (Ctrl: heathy controls; Ml: myocardial infarction patients; $* * * P<0.0001)$.
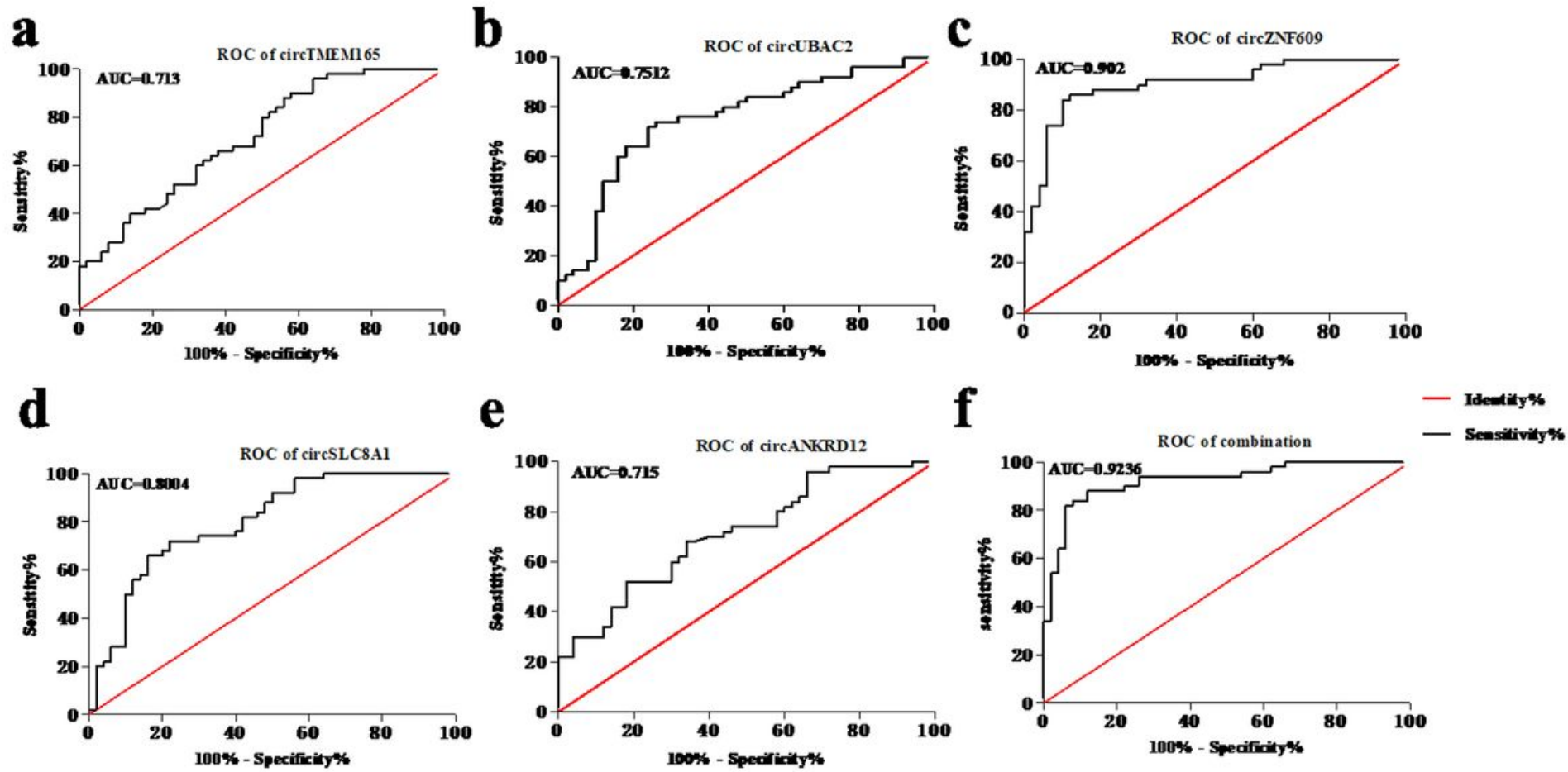

Figure 4 
Diagnostic value of RT-qPCR-validated circRNAs. Analysis of the sensitivity and specificity of circRNAs and circRNAs combination as a novel MI marker by ROC curve. (a-e) ROC curves of five RT-qPCRvalidated circRNAs. (f) ROC curves of circRNAs combination $(n=50)$.
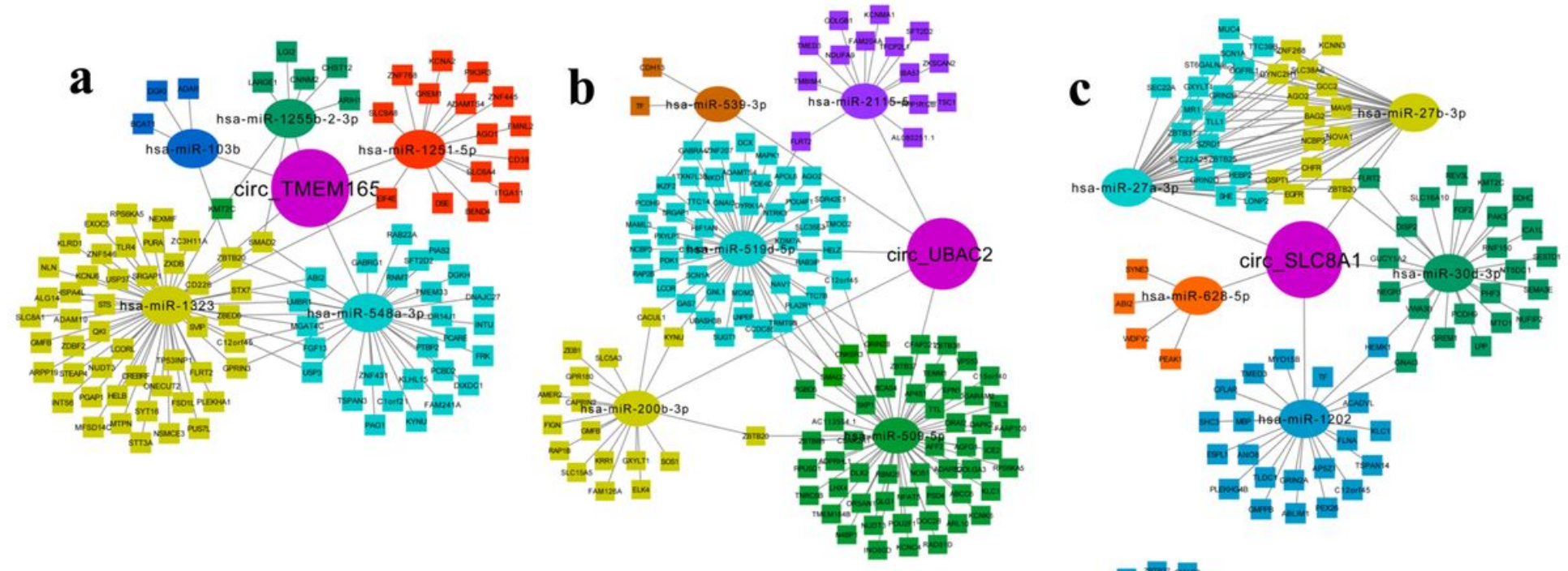

d

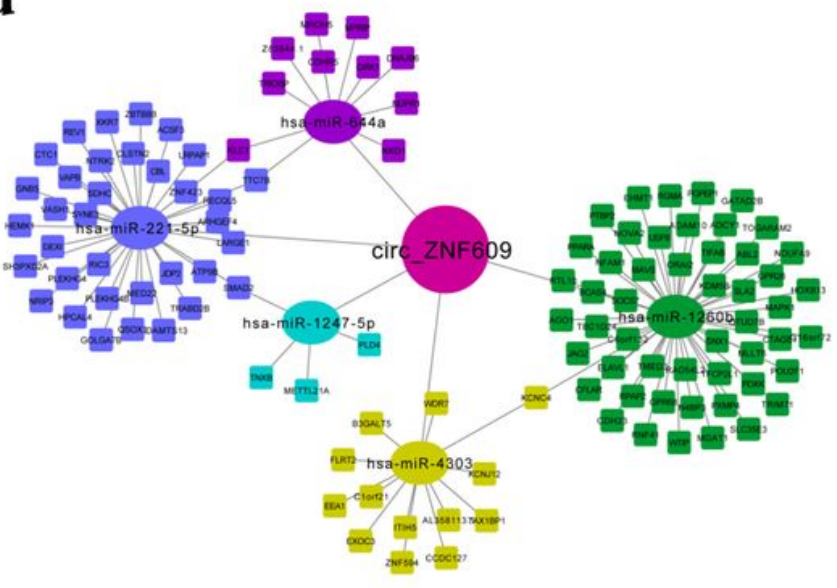

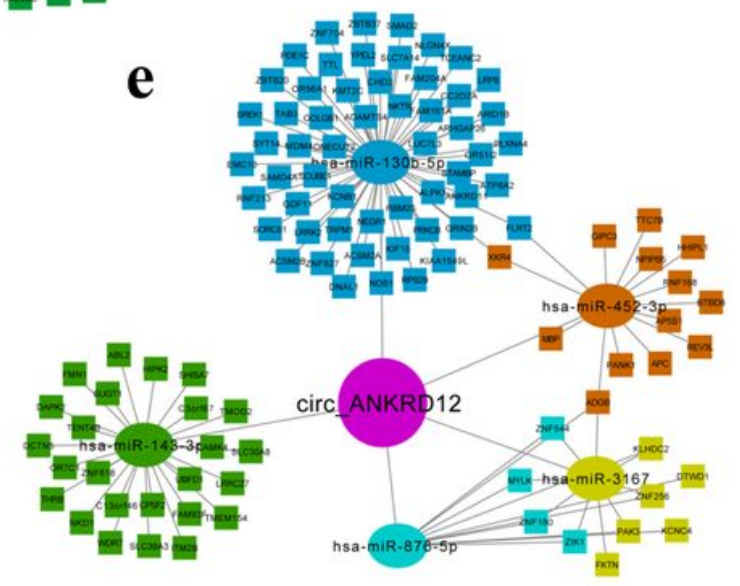

\section{Figure 5}

Predicted the network of circRNA-miRNA-mRNA. Predicted circRNA-miRNA-mRNA network for the five upregulated circRNAs in MI. (a)circ-TMEM165, (b) circ-UBAC2, (c) circ-SLC8A1, (d) circ-ZNF609, (e) circANKRD12. Circular represents circRNAs, oval represents miRNAs, and rectangle represents mRNAs. 
a

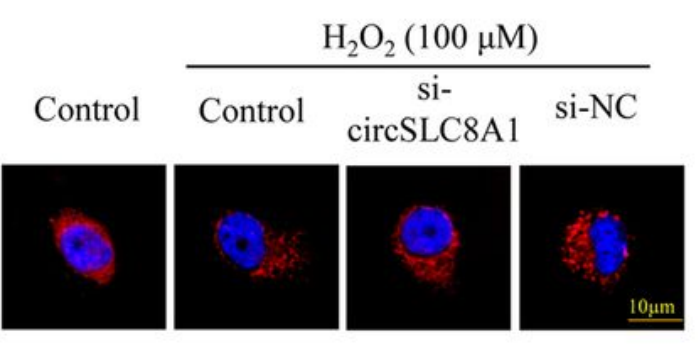

c

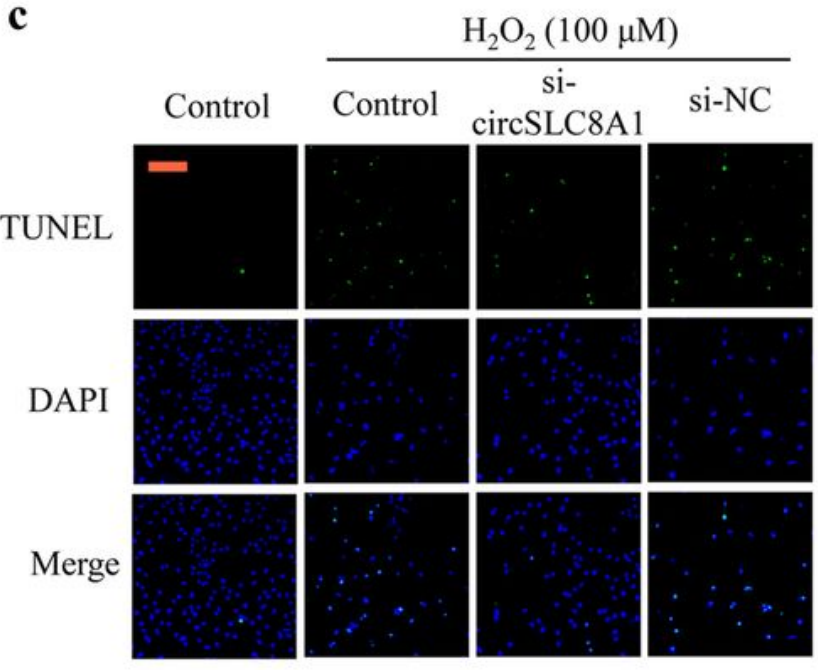

e

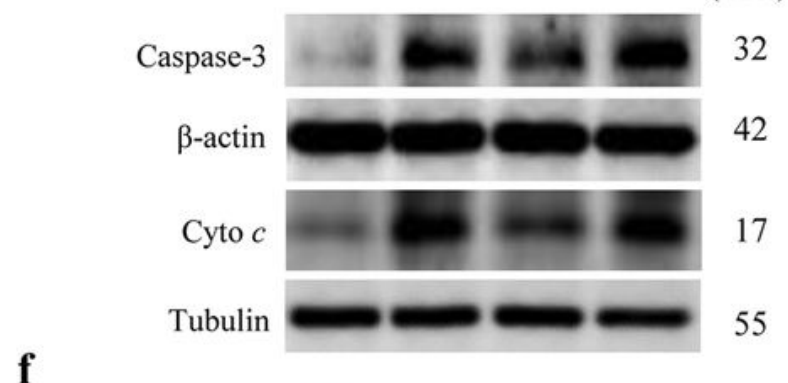

b

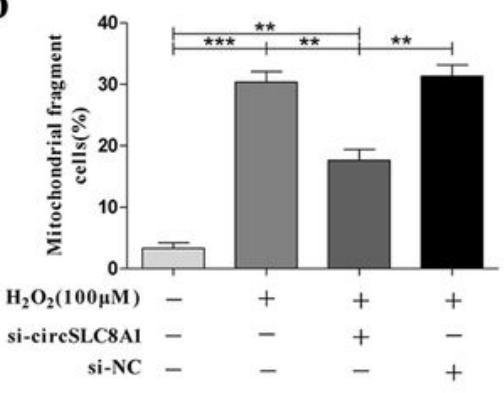

d

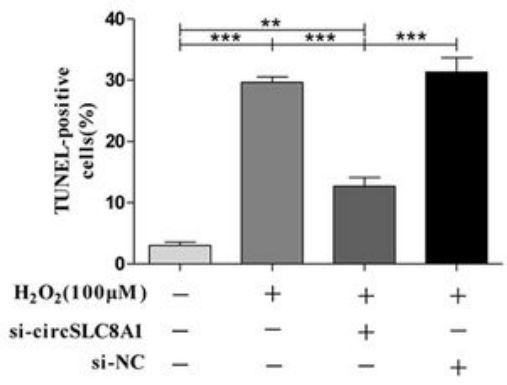

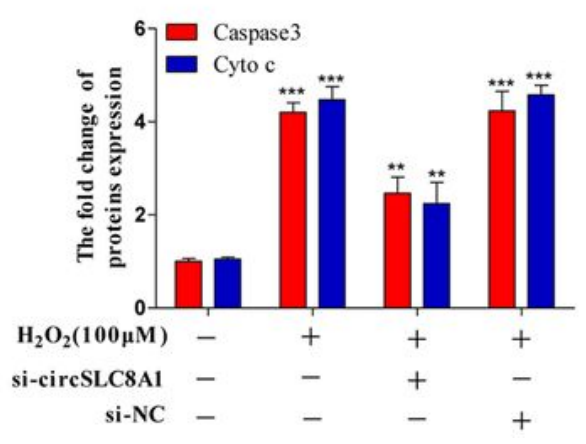

Figure 6

Knockdown circSLC8A1 protects cardiomyocytes against $\mathrm{H} 2 \mathrm{O} 2$ induced apoptosis After knockdown circSLC8A1 in cardiomyocytes, the cells were treated with $100 \mu \mathrm{M} \mathrm{H} 202$ for 12 hours. (a) Mitochondrial morphology was stained with Mito Tracker Red and observed by using a laser-scanning confocal microscope. (b) The statistical analysis of the percentage of cells undergoing mitochondrial fission. (c) Apoptotic cells were detected by TUNEL assay. (d) The statistical analysis of the percentage of apoptotic 
cells. (e) Apoptosis related protein Caspase3 and cytochrome c were detected by western blotting. (f) The statistical analysis of Caspase 3 and cytochrome $c$ relative expression levels. All of the data was expressed as the mean \pm SEM of three independent experiments. ${ }^{*} P<0.05,{ }^{\star *} P<0.01,{ }^{\star \star \star} P<0.001$.

\section{Supplementary Files}

This is a list of supplementary files associated with this preprint. Click to download.

- Supplementarydata1.xlsx

- Supplementarydata2.xlsx

- Supplementarydata3.xlsx

- SupplementaryFigure1.tif

- SupplementaryFigure3.tif

- SupplementaryFigure2.tif

- SupplementaryFigure4.tif 